\title{
HIERARCHICAL MODELING AND FINITE ELEMENT ANALYSIS OF PIEZOELECTRIC ENERGY HARVESTER FROM STRUCTURE-PIEZOELECTRIC-CIRCUIT INTERACTION
}

\author{
P.C. Ramegowda ${ }^{1}$, D. Ishihara ${ }^{1}$, R. Takata ${ }^{1}$ and T. Horie ${ }^{1}$ \\ ${ }^{1}$ Department Mechanical Information Science and Technology \\ Kyushu Institute of Technology, 680-4 Kawazu, Iizuka, Fukuoka, 820-8502, Japan
}

Key words: Hierarchical decomposition,Strongly coupled partitioned iterative method, Finite element method, Structure-piezoelectric-circuit interaction, Flexible piezoelectric energy harvesting device (FPED)

\begin{abstract}
The piezoelectric energy harvesting devices for the conversion of mechanical vibration into electric energy via a flexible piezoelectric energy harvesting (FPED) structure have gained greater attention. Here, the large deformation of the FPED structure causes a strong interaction with the electric field (direct-piezoelectric effect) and structural field (inverse-piezoelectric effect), and vice-versa. Also an electrical circuit is attached to the electrodes covering the piezoelectric layers. This becomes a three-way coupling of the structure, the electromechanical effect of the piezoelectric material, and the electrical circuit. A mathematical and numerical model of the complex physical system of the involved multiphysics coupling characteristics in order to predict the operational properties and to increase the performance is very important. The presentation will discuss a partitioned coupling algorithm based hierarchical decomposition using finite element method for piezoelectric energy harvesting from structurepiezoelectric-circuit interaction. Results obtained with the finite element analysis are compared with the experimental results of PEHDs with base excitation reported in the literature.
\end{abstract}

\section{Introduction}

Piezoelectric materials exhibit both the sensing and actuation properties because of two kinds of electromechanical coupling effects, namely, direct-piezoelectricity, inverse-piezoelectric effect, respectively. In recent years, piezoelectric materials are used in vibration-based energy harvesting from ambient vibrations $[1,2]$. Over the last decade, vibration-based energy harvesting has attracted significant research. Numerical simulation based on finite element analysis (FEA) has distinctive superiority over theoretical and experimental research. FEM not just guarantee high accuracy, but can also be used to solve complex geometric shapes, complicated multiphysics problems, and constitutive models. Comparing FE solution results with experimental results can provide a basis for the development of experimental research and theoretical models.

Tanaka et al., [1,2] proposed a type of energy harvester (see Figure 2 in [1]) termed as FPED consists of multilayer stacks of the piezoelectric film (PVDF), a soft material substrate (silicone rubber), and electrode layers attached to the external circuit. The electric circuit coupled with the piezoelectric energy harvester might include components such as a resistive load to evaluate the power output, a rectifier to convert alternating current to direct current, and a capacitor to store the harvested energy. In $[3,4]$ the electromechanical behavior of the thin cantilevered piezoelectric energy harvesters connected to a resistive load was extensively investigated using an analytical approach based on the EulerBernoulli 


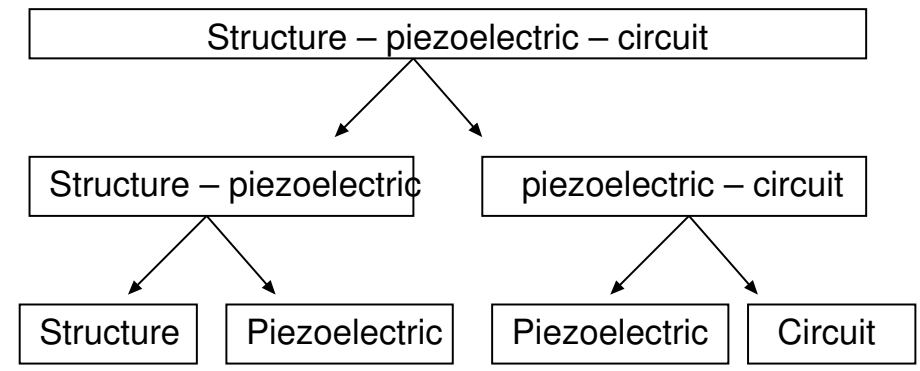

Figure 1: Hierarchical decomposition of the structurepiezoelectriccircuit interaction, where the structurepiezoelectriccircuit interaction is decomposed to the structurepiezoelectric and piezoelectriccircuit interaction interactions. Then, these subsystems are decomposed to each field.)

beam assumption. However, this approach is quite challenging for more complicated configurations, such as a composite material, complicated structural shape, and complicated electrode configuration. Therefore, numerical approaches are necessary for further investigating the electromechanical behavior of such systems.

Present work employs a novel partitioned iterative method for the structure-piezoelectric-circuit interaction using hierarchical decomposition, a partitioned iterative method for two coupled fields, and loop union. Here, the direct piezoelectric, inverse piezoelectric, and circuit solvers communicate with each other iteratively in each time step such that the coupling terms are satisfied with a high accuracy. At first, the whole system of the

systems (i.e., structure-pier pled fields are applied to t reduced to a single couple posed to each fields of can be seen repeatedly
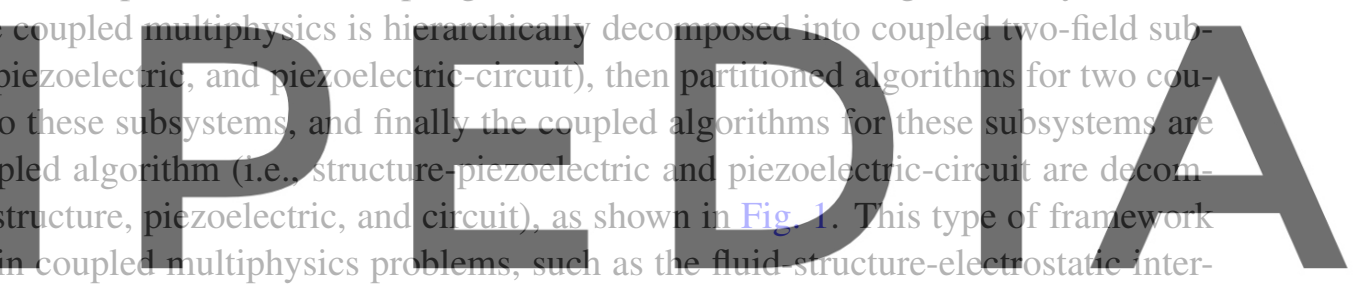
action [5] and the fluid-structure-piezoelectric interaction [6-9], as well as the structure- piezoelectric-

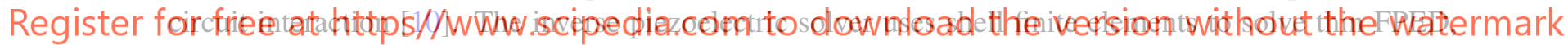
and the direct piezoelectric solver uses solid finite elements to solve an arbitrary three-dimensional distribution of the electrical potential in the piezoelectric continuum accurately $[11,12]$. The quantities are exchanged between these solvers using block Gauss-Seidel iterative methods (BGS) [13]. The electrical potential and charge within the electrode layers are unknown in the cases of an open circuit. The silicone rubber layers can be analyzed as an elastic body with electrical boundary conditions. Therefore, a pseudo-piezoelectric technique [14-16] is used to consider the electrode and silicone rubber.

\section{Governing equations of inverse-piezoelectric, direct-piezoelectric and electrical circuit}

The constitutive relation describing direct and inverse piezoelectric effects in a piezoelectric material is given by the following coupled electromechanical equations:

$$
\begin{aligned}
\sigma & =C^{E} S-e^{T} E, \\
D & =e S+\varepsilon E,
\end{aligned}
$$

where $\sigma$ and $S$ represent the stress and strain vectors, respectively; $E$ and $D$ denote the electric field and electrical displacement vectors, respectively; and $C^{E}, e$ and $\varepsilon$ represent the elasticity matrix at a 
constant electric field, piezoelectric constant matrix and dielectric constant matrix at a constant strain, respectively. The global state of the electrical circuit can be described by the global variable of the scalar electric charge $Q$. A SDOF governing equation of the electrical circuit can be derived using Kirchhoffs law as

$$
R \dot{Q}+V_{\mathrm{p}}=V_{\mathrm{e}},
$$

where $R, V_{\mathrm{p}}$, and $V_{\mathrm{e}}$ are the electrical resistance, the electric potential difference given by the piezoelectric continuum, and the electric voltage given by the external electric power supply, respectively.

\section{Finite element equations of structure-piezoelectric-circuit coupling}

The finite element discretized equations for the piezoelectric material are obtained using the standard finite element formulation for the electrostatic equilibrium from the Maxwell's equation, the mechanical equilibrium, and the constitutive relation of piezoelectricity together with the elementary and natural boundary conditions as [17]

$$
\begin{aligned}
\mathbf{M}_{\mathrm{uu}} \ddot{\mathbf{u}}+\mathbf{K}_{\mathrm{uu}} \mathbf{u}+\mathbf{K}_{\mathrm{u} \phi} \phi & =\mathbf{F}, \\
\mathbf{K}_{\mathrm{u} \phi}^{\mathrm{T}} \mathbf{u}+\mathbf{K}_{\phi \phi} \phi & =\mathbf{q}_{\mathrm{p}}+\mathbf{q}_{\mathrm{c}},
\end{aligned}
$$

where $\mathbf{M}_{\mathrm{uu}}, \mathbf{K}_{\mathrm{uu}}, \mathbf{K}_{\mathrm{u} \phi}$, and $\mathbf{K}_{\phi \phi}$ represents, respectively, the mass matrix, the mechanical stiffness matrix, the piezoelectric coupling matrix, and the dielectric stiffness matrix of the piezoelectric material;

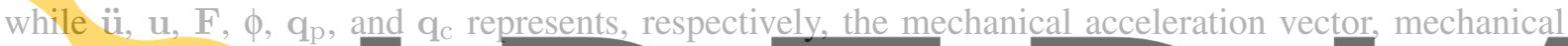
displacement vector, mech vector, and external ele T stands for the transpo
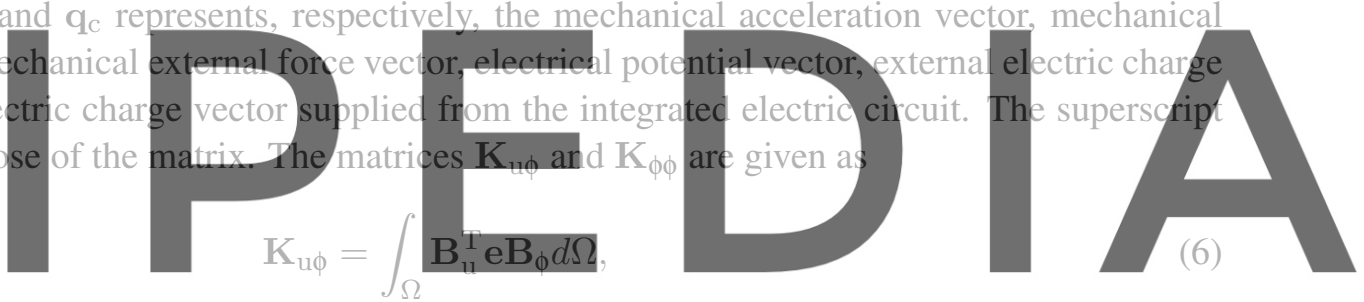

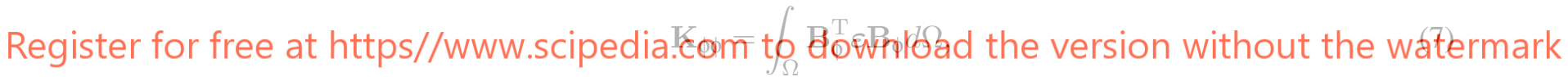

where $B_{u}$ and $B_{\phi}$ are the gradient of the interpolation functions of the displacement and the electric potential, respectively, while $\Omega$ is the three dimensional piezoelectric domain. The continuities of the electric potential and the electric charge are satisfied at the interface between the piezoelectric continuum and the electric circuit. The continuity conditions imposed on the interface can be formulated as [10]

$$
\begin{aligned}
& V_{\mathrm{p}}=\phi_{+}^{*}-\phi_{-}^{*}, \\
& \mathbf{q}_{\mathrm{c}}=\int_{s_{+}^{c}} \mathbf{N}_{\phi}\left(Q / S_{+}^{c}\right)-\int_{s_{-}^{c}} \mathbf{N}_{\phi}\left(Q / S_{-}^{c}\right),
\end{aligned}
$$

where $\phi_{x}^{*}(x$ is + or -) denotes the electric potential at the point in S xc where the circuit is connected and $\mathbf{N}_{\phi}$ is the global assemblage of the interpolation functions used for the electric potential.

\section{Pseudo piezoelectric method for electrode and silicone rubber layers}

The piezoelectric constant matrix e for a conductor (electrode) is zero, meaning that the interior of the conductor has no electric field. Therefore, the vector $\mathbf{E}$ becomes zero for a conductor layer. Hence, the 
constitutive relation describing the inverse piezoelectric-effect Eq.(1) is reduced to elastic body equation given by

$$
\boldsymbol{\sigma}=\mathbf{C}^{E} \mathbf{S}
$$

Therefore, the piezoelectric coupling matrix $\mathbf{K}_{\mathrm{u \phi}}(6)$ is given as

$$
\mathbf{K}_{\mathrm{u \phi}}=\mathbf{0} \text {. }
$$

The solid direct-piezoelectric analysis equation given in (5) for a piezoelectric material is reduced to the following pseudo direct-piezoelectric equation for the electrode and silicone rubber layers given as

$$
\mathbf{K}_{\phi \phi} \phi=\mathbf{q}
$$

Thus, Eq.(12) is considered as the finite element discretized equation for the conductor in the static electric field by taking a value large enough for each component of the electric permittivity constant matrix. The details of the coupling using the partitioned iterative method and loop union used in this paper can be found in [10].

\section{Analysis of flexible piezoelectric energy harvester}

\subsection{Problem setup}

Fig. 2 shows the FPED cantilever is under forced vibration (base excitation) setup. The length $L$ and the width $w$ of the FPED is $200 \mathrm{~mm}$ and $30 \mathrm{~mm}$, respectively, the thickness of each piezoelectric layer is $80 \mu \mathrm{m}$, the top and bottom silicon rubber layers have thickness of $0.5 \mathrm{~mm}$ each, the central rubber layer thickness is $3.0 \mathrm{~mm}$, and the electrode layer thickness is 1.0
polarized in the same directions. The forced displaced displacement
of time $t$ is given as
$u(t)_{\text {base }}=u_{0}$ sinwt.

where $\omega$ is the frequency of the base excitation, and $u_{0}$ is the forced displacement amplitude. The forced acceleration amplitude $a_{0}$ and the forced displacement amplitude are related through:

The forced displacement $u_{0}$ varies gradually in-order to avoid the numerical instability due to the sudden impact on the structure, given as

$$
u_{0}=\left\{\begin{array}{l}
\frac{u_{\max }}{2}(1-\cos \bar{\omega} t)=\frac{a_{0}}{2 \omega^{2}}(1-\cos \bar{\omega} t),(t<=\bar{t}) \\
u_{\max }=\frac{a_{0}}{\omega^{2}},(t>\bar{t})
\end{array}\right.
$$

where $\bar{\omega}=\pi / \bar{t}$ and $\bar{t}=1 /(2 f)$. The potentials of the electrode located beneath the top PVDF and the electrode above the bottom PVDF layer is grounded, while other electrodes are in an open circuit condition (potentials are unknown). In the experimental setup [2], the generated voltage is measure using a voltmeter with an internal resistance of $1.0 \mathrm{M} \Omega$. Therefore, we set the circuit load resistance same as 1.0 $\mathrm{M} \Omega$ which is an open circuit condition for this setup. The shell element is placed at the neutral axis of the FPED to solve the inverse-piezoelectric effect. The solid elements are 20-node hexahedral elements to solve direct-piezoelectric and direct pseudo-piezoelectric effect (7103 nodes and 960 elements), while the shell elements are MITC4 shell elements [18] (122 nodes and 60 elements). The FPED is harmonically excited with the constant constant acceleration $a_{0}=0.5 \mathrm{~m} / \mathrm{s}^{2}$. The time increment is fixed to $1.0 \mathrm{~ms}$. 


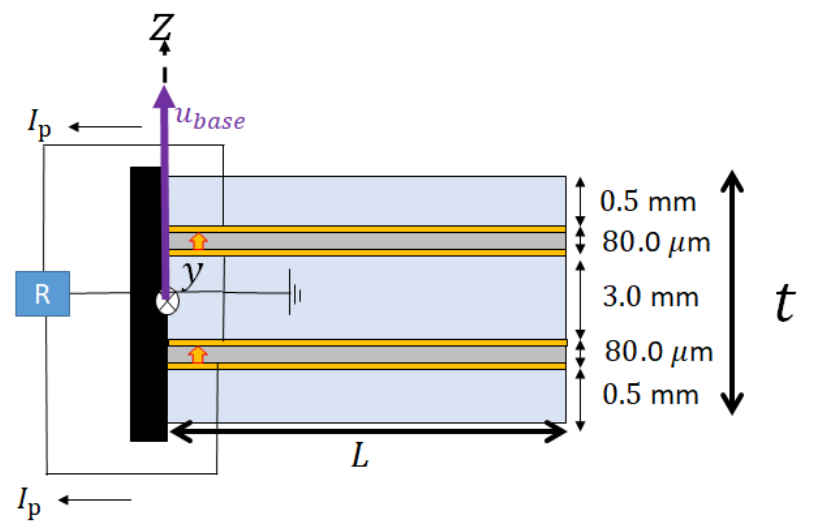

$\square$ Silicone rubber $\square$ PVDF $\square$ Electrode $\hat{0}$ polarization $R$ Resistance

Figure 2: FPED with external circuit structure subjected to forced vibration at the fixed end $(x=0)$

\subsection{Results and discussions}

Fig. 3 shows the frequency response function of the output voltages. It shows that, the natural frequency of the experiment and the simulated results are very close to each other. The natural frequency obtained

from the numerical result is $f=6.60 \mathrm{~Hz}$. The natural frequency obtained from the numerical results

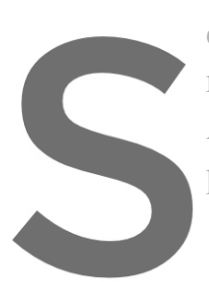
coincides with that of the

ruler tool. Also, the output

As shown in this figure, the

piezoelectric harvester and
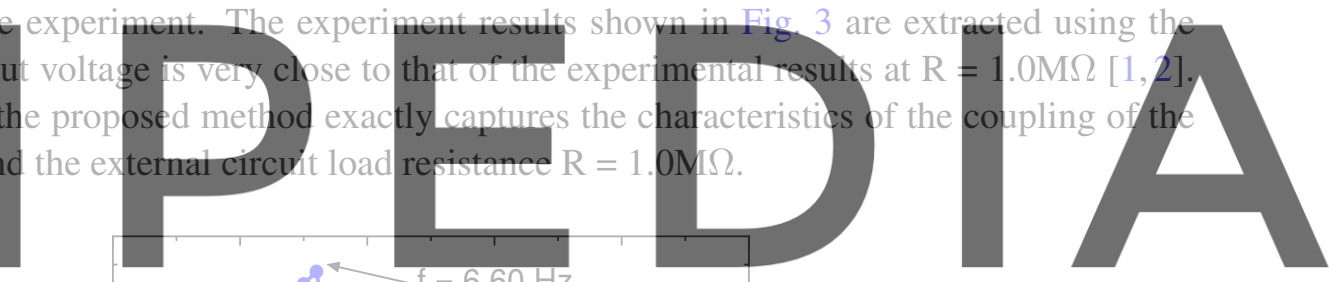

Register for free at https//www.scipedia.com to download the version without the watermark

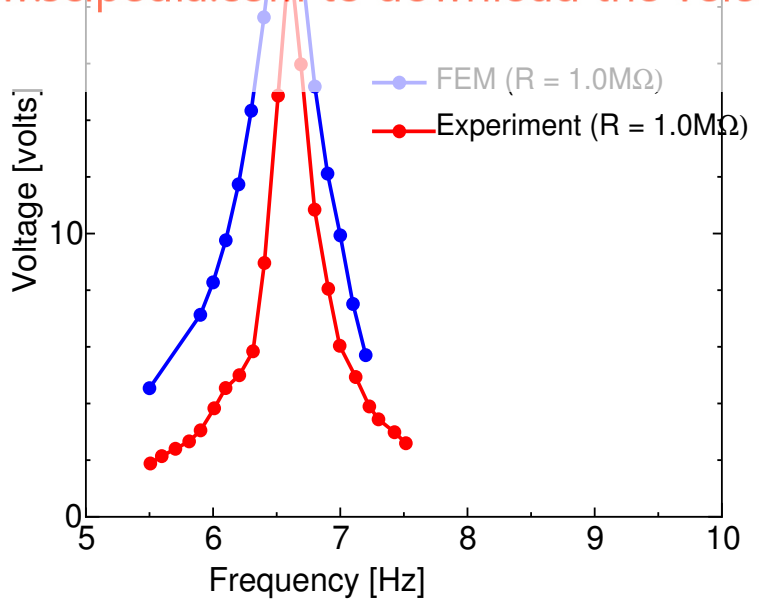

Figure 3: Output voltage v/s base acceleration excitation frequency 


\section{Conclusions}

This study presented the finite element simula- tion results of the mechanical-vibration-driven flexible piezoelectric energy harvesting device for the base excitation with mass proportional damping using solid-direct piezoelectric, shell-inverse piezoelectric and single degrees of freedom electrical circuit coupled interaction analysis method. The output voltages and displacements from the numerical simulations are close to the experimental results conducted. Comparing FE solution results with experimental results provide a basis for the development of experimental research.

\section{Acknowledgments}

This research was supported by the Japan Society for the Promotion of Science, KAKENHI Grant Nos. 17H02830, 19F19379, and 20H04199. We would like to thank Prof. Y. Tanaka at Hiroshima university for his helpful discussion.

\section{REIERENCES}

[1] Tanaka, Y. Matsumura, K. and Mutsuda, H, Study on flexible power generation device using piezoelectric film, Journal of Energy and Power Engineering. 6 (2012) :353-360.

[2] Tanaka, Y. Oao, T. Mutsuda, H Popov, A.A. Pater, R. and McWilliam, S, Forced vibration experiments on flexible piezoelectric devices operating in air and water environment, International Journal of Applied Electromagnetics and Mechanics. 45 (2014) :573-580.
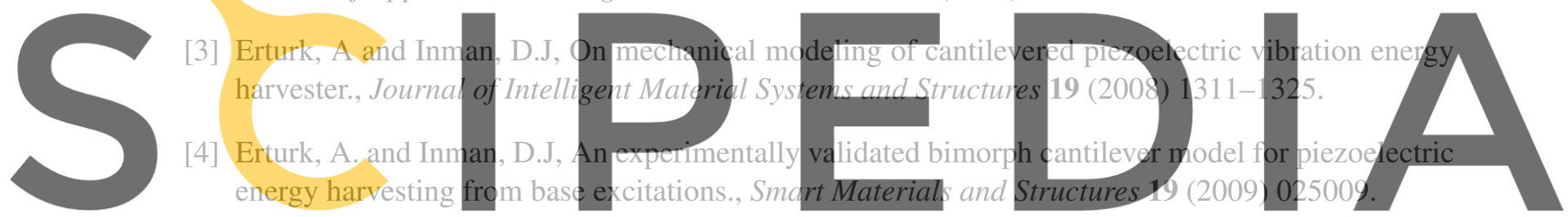

[5] Ishihara, D. Horie, T and Niho, T, Hierarchal decomposition for the structure-fluid-electrostatic

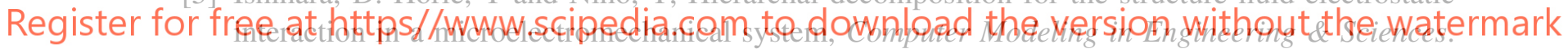
108 (2015) :429-452.

[6] Ramegowda, P.C. Ishihara, D. Takata, R, Niho, T. and Horie, T, Hierarchically decomposed finite element method for a triply coupled piezoelectric, structure, and fluid fields of a thin piezoelectric bimorph in fluid, Computer Methods in Applied Mechanics and Engineering. 365 (2020) :113006.

[7] Ramegowda, P.C. Ishihara, D. Takata, R. Niho,T. and Horie, T, Fluid-structure and electric interaction analysis of piezoelectric flap in a channel using a strongly coupled fem scheme, Proceeding of the 6th European Conference on Computational Mechanics (ECCM 6 2018), Glasgow. (2018) :382-393.

[8] Ramegowda, P.C. Ishihara, D. Takata, R. Niho,T. and Horie, T, A finite element approach for a coupled numerical simulation of fluid -structure-electric interaction in mems., Proceeding of the VII International Conference Computational Methods for Coupled Problems In Science and Engineering, Greece. (2017) :999-1007. 
[9] Ramegowda, P.C. Ishihara, D. Takata, R. Niho,T. and Horie, T, Triply coupled analysis method for thin flexible piezoelectric bimorph in fluid., Proceeding of the VIII International Conference Computational Methods for Coupled Problems In Science and Engineering. Spain (2019) :470457.

[10] Ishihara, D. Takata, R. and Ramegowda, P.C., Partitioned iterative method for inverse piezoelectricdirect piezoelectric-electric circuit interaction, Transactions of the Japan Society for Computational Engineering and Science. 2020 (2020): 10 pages.

[11] Ramegowda, P.C. Ishihara, D. Niho, T. and Horie, T, A novel coupling algorithm for the electric fieldstructure interaction using a transformation method between solid and shell elements in a thin piezoelectric bimorph plate analysis, Finite Elements in Analysis and Design. 159 (2019) :33-49.

[12] Ramegowda, P.C. Ishihara, D. Takata, R. Niho,T. and Horie, T, A novel coupled algorithm for shell structure-electric interaction analysis in mems piezoelectric actuator., Proceedings of The 35th JSST Annual Conference International Conference On Simulation Technology, Kyoto. (2016) :332-339.

[13] Ramegowda, P.C. Ishihara, D. Niho, T. and Horie, T, Performance evaluation of numerical finite element coupled algorithms for structure-electric interaction analysis of mems piezoelectric actuator, International Journal of Computational Methods. 16 (2019) :1850106.

[14] Ramegowda, P.C. Ishihara, D. Takata, R, Niho, T. and Horie, T, Finite element analysis of a

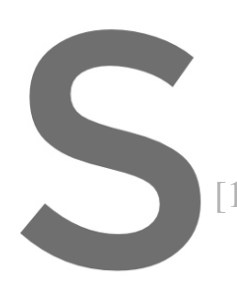
thin piezoelectric bimorph with a metal shim using solid diret
piezoelectric coupling with psetde direct-piezoclectric evaluate
(2020) :112284.
Ishihara, D. Ramegowda, P.C Takata, R. Niho.T. and Horie, T,
shell inverse-piezoelectric analysis using partitioned method for metal layers., Proceeding of the VIII International Conference C
Problems In Science and Engineering. Spain (2019):451-4.57.

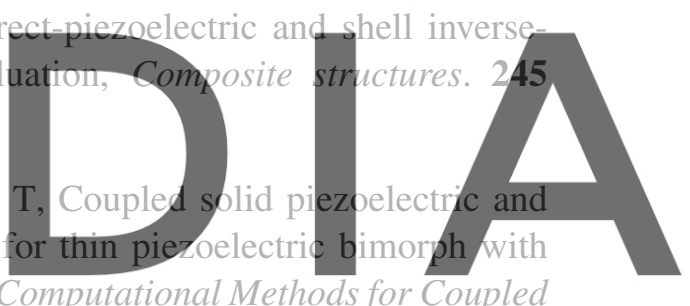

Register for free at https//WWW.SCIpedia.Com to downiload the version without the watermark

[16] Takata, R. Ishihara, D. Ramegowda, P.C. Niho, T. and Horie, T, Solid piezoelectric-shell inverse piezoelectric partitioned analysis method for thin piezoelectric bimorph with conductor layers, Transactions of the Japan Society for Computational Engineering and Science. 2019 (2019) :20190011.

[17] J. Fish, W. Chen, Modeling and simulation of piezocomposites, Computer Methods in Applied Mechanics and Engineering; 192 (2003) :3211-3232.

[18] Noguchi,H. and Hisada, T, Sensitivity analysis in post buckling problems of shell structures., Computers \& Structures. 47 (1993):699-710. 